\title{
PRÓLOGO A LA ANTOLOGÍA DE THEOTONIO DOS SANTOS ${ }^{1}$
}

Mónica Bruckmann²

\author{
Espero que não sofras mais \\ Terras e mares \\ Suaves montanhas \\ Doces paisagens \\ cobertas de sangue \\ Formosos cenários de lutas infernais \\ Imenso caldeirão \\ onde fervem as paixões \\ de tantos e tantos povos \\ por séculos e séculos enfrentados \\ na franja de impérios colossais \\ Massa concentrada de emoções \\ Ódio e amor em estranho contubérnio. \\ Homem/mulher \\ Armas/morte \\ Sangue/terra \\ Pátria/luta. \\ Dura sobrevivência \\ Pátria - luta - mundo \\ imensos ideais aqui ficam cultivados \\ à busca da paz duradoura \\ para toda a humanidade
}

\footnotetext{
${ }^{1}$ Prólogo de la Antología de Theotonio dos Santos: Construir soberanía, una interpretación económica de y para América Latina (Volumen I), publicada por el Consejo Latinoamericano de Ciencias Sociales - CLACSO, en noviembre de 2020.

${ }^{2}$ Doctora en Ciencia Política y profesora del Departamento de Ciencia Política y del Programa de Posgrado en Historia Comparada de la Universidad Federal de Río de Janeiro (UFRJ). Email: monicabruckmann@gmail.com
} 
não pode garantir a paz

para o próprio povo

ferido de morte

nas suas próprias entranhas

Quisera estabelecer a tolerância

das raças, dos povos,

religiões, línguas e pátrias

sonho de uma só nação unificada

pela resistência aos opressores

Aqui se formou a vitoriosa

ambição do não alinhamento

que derrubou impérios colossais

altiva cabeça erguida contra os

poderosos

Eis que a construção de paz

se rue sob as armas do terror

Feridas profundas

ódios ancestrais

que os poderosos souberam açular

Braças revolvidas pelas ambições

Pelos desejos de expansão

detidos pela indomável

e generosa coragem

dos que lutaram por seus sonhos

Auto-governo

Auto-nomia

Auto-gestão

dos povos e dos indivíduos

livres e solidários

Aqueles que conheci em

colóquios intermináveis.

(DOS SANTOS, 2002)

Esta antología reúne una selección de textos de la obra científica de Theotonio dos Santos, publicada entre 1962 y 2016. No fueron incluidos los textos literarios, entre los cuales puedo destacar el libro de poemas Construção (1957) y Oferendas: Um poema-oração para Yemanjá e para a humanidade inteira (inédito), así como la ópera en homenaje al Che Guevara titulada "Che, ópera pop latinoamericana". Tampoco se incorporaron en esta publicación los artículos de crítica literaria publicados en periódicos y revistas en la época juvenil, iy algunos escritos de lo que sería su libro de memorias, que no llegó a concluir y que llevaría como título "Eu topo!”, cuya 
traducción más aproximada al español es: ¡Estoy dispuesto! El autor dejó una vasta producción periodística que se inicia precozmente a los 15 años (1951/52) cuando funda el semanario A Voz Juvenil y que mantuvo a lo largo de la vida como columnista de varios periódicos en Brasil y América Latina, entre los que destacan los suplementos culturales el Diario de Minas y Folha de Minas, o medios de gran circulación como Jornal do Brasil, El Universal y Reforma, de México.

Es poco conocido el hecho de que Theotonio inicia su vida intelectual como poeta y ensayista literario a través de una participación intensa en el ambiente cultural de Belo Horizonte (Minas Gerais), durante los años 50. Con 19 años formó parte de un movimiento cultural conocido como "Generación Complemento", que debía su nombre a la revista literaria "Complemento" que fundó junto a otros jóvenes intelectuales de Minas Gerais. De este movimiento participaron literatos, pintores, críticos de arte y bailarines entre los cuales se destacaron Klaus y Ángel Viana, que contribuyeron a la creación de una nueva estética en la danza moderna brasileña, el escritor Silviano Santiago, el crítico de arte Frederico Morais, la pintora Vilma Martins, el productor musical Ezequiel Neves, entre otros jóvenes de espíritu inquieto y contestatario.

La generación complemento se convirtió en un movimiento cultural de proyección nacional, que propuso una crítica vehemente a los patrones estéticos dominantes y abordó un debate filosófico y ético desde una perspectiva humanista que revelaba una gran admiración por el existencialismo filosófico. De alguna manera, se convirtió también en un núcleo de formación de intelectuales que tuvieron gran impacto en la producción cultural brasileña.

El primer libro de Theotonio, publicado en 1957 a los 21 años, es un poemario titulado $A$ construção, que tuvo una gran acogida por la crítica literaria y abrió un debate importante en torno a su estilo y contenido. El poemario ya evidenciaba una preocupación social y una visión política, como vemos en este trecho a continuación: 
Os operários vieram do nada

Seus seios de pedra, suas mãos incrustradas

No fundo das cores.

Os operários,

Os primeiros e últimos caminhantes

Da poesia construída entre os galhos.

Líquidos como as aves,

Líquidos.
Antes se contorceram
Em busca da dor,
Até que tudo se transformou
Na primeira pedra.
o gelo, as distancias.
o branco revoar dos ódios.
Os operários vieram do nada
E nada encontraram.

(DOS SANTOS, 1957)

A partir de la incursión en la vida cultural de Belo Horizonte y su participación en el debate sobre una nueva propuesta estética, ética y filosófica, Theotonio va a iniciar su vida intelectual, asumiéndose como intelectual orgánico, actitud que mantuvo a lo largo de la vida. Cuatro décadas después, a los 65 años, escribirá en su diario:

Aos 65 anos, vivo um momento dramático. Hora de decisões. De um tardio amadurecimento. Não posso dizer que mina vida seja um barco oscilante sobre as ondas da história. Lutei muito para fazê-la, influenciá-la, direcionála.

Tomei o lado certo na história: o dos trabalhadores, produtores do futuro, como sociedade, economia e cultura. Mas a luta de classes é uma guerra secular com muitas derrotas e poucas vitorias.

Assim foi com a revolução burguesa. Desde o século XV a burguesia vislumbrava a possibilidade de uma nova sociedade, uma nova economia, uma nova cultura, uma nova civilização (DOS SANTOS, 2002).

En 1958 inicia su formación como científico social en la facultad de Sociología, política y administración de la Universidad Federal de Minas Gerais (UFMG). Podríamos decir que Brasil perdió un poeta, pero ganó un científico social que se dedicó al estudio de la formación económica y social brasileña y del proceso latinoamericano en el contexto de las reconfiguraciones del sistema mundial y del capitalismo contemporáneo.

La obra de Theotonio dos Santos expresa la densidad del pensamiento social latinoamericano y representa uno de los referentes teóricos más 
rigurosos y creativos del pensamiento marxista contemporáneo. Como diría el economista chileno-mexicano José Valenzuela Feijoo: el pensamiento de Theotonio es tan vasto y creativo, que a veces parece superar la infinita realidad.

El autor forma parte de una corriente de pensamiento latinoamericana que se apropió del marxismo como una matriz teórica universal para analizar la realidad local y producir nuevo conocimiento y nueva teoría. Como proponen algunos autores al referirse al intelectual boliviano René Zavaleta, se trata de una nacionalización del marxismo, es decir, de un proceso de apropiación del marxismo como matriz teórico-metodológica universal que se convierte en una forma de ver el mundo y, al mismo tiempo, de analizar las realidades históricas concretas, produciendo nuevo conocimiento y nueva teoría. Esta nueva teoría, ciertamente, tiene la capacidad de aportar a la matriz teórica más general, enriqueciéndola y contribuyendo a profundizar su capacidad y densidad analítica. La teoría de la dependencia, y particularmente su vertiente marxista, significó esta posibilidad de construir nueva teoría y un nuevo enfoque del capitalismo contemporáneo desde América Latina y, al mismo tiempo, desde el análisis del proceso histórico de la región integrado a la dinámica de acumulación en los centros de la economía mundial.

Existen ya muchos estudios importantes sobre la Teoría Marxista de la Dependencia (TMD) que buscan hacer un balance de la trayectoria intelectual y la obra teórica de los primeros formuladores de esta corriente de pensamiento: Ruy Mauro Marini, Vania Bambirra y Theotonio dos Santos. Esta creciente producción es resultado de una agenda de pesquisa cada vez más sistemática de nuevas generaciones de investigadores que han multiplicado los espacios de reflexión sobre el tema en toda la región. No pretendo, en este prólogo, realizar un análisis sistemático de la producción científica de Theotonio dos Santos, sino ofrecer algunas claves de interpretación sobre las varias dimensiones de su obra. Más que un estudio acabado presentaré de un testimonio de quien compartió con él una agenda intelectual, académica y política a lo largo de casi 20 años. 
Desde esta perspectiva, presento su obra a partir de cuatro momentos analíticos y de elaboración teórica, que no necesariamente acompañan un orden cronológico, pero, ciertamente, se van acumulando y adensando: 1. La apropiación del marxismo; 2. La dinámica de la dependencia; 3. Revolución científico-técnica y economía mundial; 4. Sistema mundial y proceso civilizatorio.

\section{La apropiación del marxismo}

Entre 1960 y 1964, Theotonio inicia de manera sistemática sus estudios de marxismo. En su memorial intelectual, preparado para un concurso de profesor titular en 1994, hace un balance detallado de estos estudios, que inicia con los principales escritos filosóficos de Marx y Engels y continúa con una lectura atenta de los textos políticos, antes de debruzarse sobre el conjunto de la obra económica, desde "Salario, precio y ganancia" hasta El Capital, que fue objeto de tres años de lectura individual y dos grupos de estudio: el primero en la Universidad de Brasilia, cuando realizaba su maestría a inicios de la década de 1960 y el segundo en Chile, durante el periodo de exilio, con la participación de un amplio grupo de jóvenes intelectuales latinoamericanos, como Aníbal Quijano, Fernando Henrique Cardoso (con quien tendría debates y discrepancias profundas, años más tarde), Francisco Weffort, Pedro Paz, Vania Bambirra, entre otros. Hacia fines de la década de 1960, Chile se convirtió en un centro de confluencia de intelectuales de izquierda, que venían de diversas experiencias políticas en la región y dio origen a un gran movimiento de estudios de El Capital y del pensamiento marxista clásico y contemporáneo.

Sin embargo, los estudios de Theotonio se situaban en un debate más amplio del marxismo como corriente histórica, teórica y filosófica buscando establecer un diálogo en el campo de las ciencias sociales, la historia y la política, e incorporando el estudio del movimiento obrero y de las revoluciones sociales modernas. Emprendió una lectura sistemática de los marxistas clásicos: Kautsky, Rosa Luxemburgo, Plejanov, Lenin, Trotsky, Bujarin, Preobrazhensky, sin dejar de lado a los pensadores de la llamada 
"corriente revisionista", como Berstein, Hilferding o Adler. Se aproxima al pensamiento de Gramsci en los años 50, a partir de sus trabajos sobre los consejos obreros de la segunda década del siglo XX y de la recuperación que hicieron la escuela italiana y argentina del autor en la década de 1960, como pensador de lo nacional-popular. No obstante, siempre fue crítico del redescubrimiento posterior que se hizo de Gramsci, que lo colocaba como referencia de un "revisionismo filosófico del marxismo".

Theotonio tenía una gran disciplina al organizar sus estudios teóricos y filosóficos, que detallaba en minuciosos planes de trabajo registrados en cuadernos y fichas de lectura que lo acompañaron a lo largo de varios exilios. De tal manera que, al finalizar su formación universitaria, había conseguido acumular un bagaje importante de conocimiento del pensamiento marxista mundial y el pensamiento social brasileño. Su formación marxista estuvo acompañada de un estudio profundo de la realidad brasileña, cuya literatura conocía ampliamente Leyó completamente la obra de Caio Prado Junior, Celso Furtado, Roberto Simonsen y Nelson Sodré, de quien fue alumno durante sus estudios de maestría.

En su memorial intelectual de 1994, declara: "Ao terminar meus estudos de graduação e mestrado, já podia me considerar realmente um marxista, no sentido de que minha formação teórica em Marx e Engels era quase completa e em geral me identificava com a sua démarche teórica" (DOS SANTOS, 1994).

Los primeros trabajos que Theotonio publica forman parte de ese proceso de aproximación y apropiación del marxismo, lo que constituye, desde nuestro punto de vista, un primer núcleo de análisis y formulación teórica en la obra del autor. En 1962, con 26 años, publica el libro Quienes son los enemigos del pueblo, que tuvo como base su tesis de maestría sobre las clases sociales en Brasil, en la que analizaba las clases sociales desde una perspectiva histórica y particularmente, las clases dominantes. Este libro presenta los primeros estudios de una línea analítica que profundizará a lo largo de su producción científica, que es el imperialismo como fenómeno mundial y el antimperialismo como respuesta política en un espacio de 
articulación y dominación global. El impacto de estos dos fenómenos: imperialismo y antiimperialismo, en la política latinoamericana y particularmente en la política brasileña, se fortalecen con el análisis del latifundio, del sector financiero, de los monopolios y de la gran industria en Brasil.

Los estudios sobre el desarrollo surgen en la agenda internacional durante los años de 1950, con la consolidación de la hegemonía de Estados Unidos en la economía mundial y como consecuencia de este proceso que coloca el desarrollo en este país como un modelo global a ser seguido, a través de la idea de modernización. En sus primeros estudios, Theotonio ya plantea los límites y las contradicciones de este modelo, por su carácter excluyente y concentrador:

Durante los últimos siete años se defendió la idea, defendida particularmente por el expresidente Juscelino Kubitschek, de que la situación brasileña solo se resolvería a través del desarrollo económico que realmente llegara a todo el pueblo. La práctica mostró, sin embargo, que, a pesar de los aspectos positivos, el desarrollo que se consiguió en Brasil no fue, definitivamente, para todos. Nadie puede ser contrario al desarrollo, mucho menos el trabajador. El pueblo debe apoyar el desarrollo, pero debe luchar para orientarlo a su favor. Esto significa que el pueblo es contrario al desarrollo capitalista, al desarrollo "a cualquier precio", es decir, el desarrollo que causa hambre y miseria a las mayorías. El desarrollo que lleva siempre a las viejas posturas de "ajustar el cinturón" de los trabajadores y abrir el de los grandes capitalistas. ¿Cuáles son los efectos negativos de ese desarrollo? (DOS SANTOS, 1962, pp. 19-20).

Estos análisis van a conducir a una conclusión política fuerte: ¿En ese contexto histórico, cuáles son las fuerzas progresistas brasileñas y quienes son los enemigos del Pueblo?

Posteriormente publicó el Concepto de clases sociales, que fue la continuación de sus investigaciones durante la maestría y producto de un seminario sobre el mismo tema desarrollado en Chile, entre 1966-67. El libro fue publicado en 1973, cuando ya era director del Centro de Estudios Socioeconómicos (CESO), en Chile.

En este estudio, Theotonio profundiza su comprensión del marxismo revelando un análisis muy sofisticado y un esfuerzo por reconstruir, desde el concepto de lucha de clases, los elementos que condicionan la consciencia de clase a partir de una visón dialéctica entre ser social y consciencia social, 
menos como determinantes en una relación mecánica y más como producto de las contradicciones del desarrollo de las fuerzas productivas, en un debate muy fuerte con lo que era la visión del marxismo oficial de influencia estalinista y desde una interpretación teórica y conceptual de la propia obra de Marx. Veamos esta afirmación en las palabras del autor:

La lucha de clases está relacionada directamente con la superación de una determinada formación social (modo de producción, más modo político y cultural). De esta manera, solo se puede comprender el concepto en el contexto de las contradicciones y leyes de desarrollo interno de un determinado modo de producción y de una determinada formación social. En este nivel del análisis se integra el concepto de conciencia de clase. (...) Una de las conquistas básicas de la ciencia social marxista se define en la frase del prólogo a la Contribución a la Crítica de la Economía Política: "Y del mismo modo de que no podemos juzgar tampoco a estas épocas de revolución por su conciencia, sino que, por el contrario, hay que explicar esta conciencia por las contradicciones de la vida material, por el conflicto existente entre las fuerzas productivas sociales y las relaciones de producción". Se trata de mostrar las formas de conciencia antagónicas posibles que corresponden a determinados modos de producción (DOS SANTOS, 1973, p. 28).

Estos primeros trabajos ya muestran lo que será una característica de la obra del autor: una vocación analítica vigorosa y una visión política que se desprende de la primera. Estas dos dimensiones, intelectual y política, marcaron y articularon su obra desde los primeros escritos hasta el último libro, Desarrollo y Civilización, publicado por la Universidad del Estado de Río de Janeiro (UERJ) y el Consejo Latino Americano de Ciencias Sociales (CLACSO), en 2016.

\section{La teoría de la dependencia}

En 1962, Theotonio es invitado por Darcy Ribeiro a la Universidad de Brasilia, cuando estaba siendo creada en la nueva capital de Brasil. Es un proyecto muy interesante porque representaba una ruptura de ciertos paradigmas de la universidad brasileña. Darcy convocó jóvenes recién graduados de varias universidades del país para continuar sus estudios de posgrado y, al mismo tiempo, desempeñar tareas de docencia e investigación en la Universidad de Brasilia. Así consigue un grupo de jóvenes brillantes, becarios dedicados a tiempo completo a la vida académica y desempeñando el doble papel de estudiantes de posgrado y profesores universitarios. 
Es tal vez en este momento que se forma el primer núcleo de la teoría de la dependencia, particularmente de la vertiente marxista o neo-marxista ${ }^{3}$ que será conocida posteriormente como la teoría marxista de la dependencia (TMD). Nos referimos al encuentro intelectual entre Ruy Mauro Marini, Vania Bambirra y Theotonio, además de André Gunder Frank, economista alemán formado en la Escuela de Chicago y luego uno de los críticos más mordaces de los Chicago boys. Gunder Frank fue, por algunos meses, profesor visitante de la UNb por invitación de su rector Darcy Ribeiro.

Eran debates acalorados e intensos entre el profesor alemán y los jóvenes investigadores que luego se constituirán en los formuladores de la teoría de la marxista de la dependencia. Este proceso será abruptamente interrumpido por el golpe de 1964 en Brasil. Theotonio solo conseguirá salir de Brasil en 1966, después de dos años de clandestinidad en San Pablo. Llega como exiliado a Chile con su compañera de formulaciones teóricas y esposa en esa época, Vania Bambirra y la primera hija de la pareja, Nadia, con dos años. Chile vivía un proceso de efervescencia política y gran avance de las fuerzas populares que llevaron a Salvador Allende y la Unidad Popular al gobierno, en 1970.

Será en Chile donde el enfoque de la dependencia va a conseguir un alto nivel de formulación teórica y un desarrollo más amplio que atrajo un grupo importante de jóvenes investigadores. De esta manera se constituyó el primer núcleo de la teoría de la dependencia, que luego se convertirá en una corriente de pensamiento en América Latina. Este núcleo se articulaba en el Centro de Estudios Socio Económicos (CESO) de la Universidad de Chile, que Theotonio dirigió. En 1967 se crea un equipo de investigación sobre dependencia económica de América Latina, cuyos seminarios abordaron una amplia problemática dentro del nuevo enfoque de la dependencia, en pleno

\footnotetext{
${ }^{3}$ En el libro Teoría de la dependencia: balance y perspectivas (1973/2003) Theotonio toma la clasificación elaborada por André Gunder Frank de las diferentes corrientes de la teoría de la dependencia, a partir de los autores más citados en el debate y de sus respectivos orígenes teóricos. Así, se delinean tres corrientes: reformistas no marxistas, marxistas y neomarxistas. En los dos últimos grupos (marxistas y neo-marxistas) aparecen relacionados autores como Paul Baran, André Gunder Frank, Ruy Mauro Marini, Theotonio dos Santos, Vania Bambirra, Aníbal Quijano, Franz Hinkelammert, Emmanuel Wallerstein, Samir Amin y Warren.
} 
proceso de gestación. Del CESO formaron parte Orlando Caputo, Roberto Pizarro, Tomás Vasconi, Marta Harnecker, Marco Aurélio García, Emir Sader, Pio García, Clarisa Hardy, Alejandro Scherman, Cristina Hurtado, Cristóbal Kay, Álvaro Briones, Guillermo Labarca, Laureano Ladrón de Guevara, Manuel Lajo, entre otros. Además de Ruy Mauro Marini, Vania Bambirra y André Gunder Frank.

Este primer núcleo de la teoría de la dependencia va a desarrollar un diálogo muy intenso con gran parte de la intelectualidad de izquierda latinoamericana que circulaba por Chile en estos años, como el sociólogo peruano Aníbal Quijano, quién será uno de los formuladores de la teoría de la colonialidad en América Latina y un interlocutor y también formulador de la dependencia.

Estos análisis se desarrollan al calor del momento político y del potencial transformador del gobierno de Salvador Allende e influenciaron fuertemente el programa de la Unidad Popular. La teoría de la dependencia siguió un camino crítico a las formulaciones de las teorías del desarrollo económico que proponían un proceso lineal de transformación de las economías subdesarrolladas en economías desarrolladas, a partir de una cierta idea de modernización. El enfoque novedoso de la teoría de la dependencia consistió en mostrar que el camino del desarrollo económico en Europa, Estados Unidos o Japón no era viable para las economías latinoamericanas, pues el subdesarrollo en estos países, al igual que en los países poscoloniales de África y Asia, no era resultado de la conservación de economías precapitalistas, sino de la forma en que estas economías se habían integrado a la economía mundial.

La teoría de la dependencia buscó comprender el capitalismo dependiente como proceso orgánico a la formación de una economía capitalista mundial, mostrando que los cambios en la formación de una nueva dependencia en América Latina estaban articulados a los cambios en el centro del sistema, especialmente en Estados Unidos.

En su libro Teoría de la dependencia, balance y perspectivas, Theotonio recoge cuatro puntos propuestos por los economistas suecos Magnus 
Blomstron y Bjorn Hettne como resumen las ideas centrales de la teoría de la dependencia: 1 . El subdesarrollo está conectado de manera estrecha con la expansión de los países industrializados; 2. El desarrollo y el subdesarrollo son aspectos diferentes de un mismo proceso universal; 3. El subdesarrollo no puede ser considerado como primera condición para un proceso evolucionista; 4. La dependencia no es solo un fenómeno externo, sino que se manifiesta también bajo diferentes formas en la estructura interna, social, ideológica y política.

Los análisis de la dependencia muestran que el subdesarrollo no es un fenómeno aislado ni endógeno, sino parte de un proceso orgánico a la dinámica de acumulación de los países industrializados y de los centros de la economía mundial. Es decir, desarrollo y el subdesarrollo son dos aspectos diferentes de un proceso único universal: el capitalismo contemporáneo. Desarrollo y subdesarrollo son dos caras de la misma moneda ya que los centros más dinámicos de la economía mundial necesitan de una periferia subdesarrollada, exportadora de materias primas o productos con el menor valor agregado posible para viabilizar su modelo de acumulación.

Esta es una de las críticas más fuertes que la teoría de la dependencia formula a las teorías del desarrollo que surgen en la segunda mitad del siglo XX y que tenían en la CEPAL su principal centro de formulación teórica. Muestra que el pensamiento de Raúl Prebisch y los demás pensadores de CEPAL, entre los años de 1950 y 1960, estaba inscrito en el paradigma de la modernización, al proponer como objetivo la superación de los obstáculos nacionales e internacionales que inviabilizaban el desarrollo económico y que impedían la transición hacia una economía capitalista y a la posibilidad de beneficiarse de los "frutos del progreso tecnológico".

De hecho, la teoría de la dependencia impactó en el análisis de los fenómenos estudiados por la teoría del desarrollo, integrando nuevas problemáticas como la estructura de clase, el fenómeno de la marginalidad y los nuevos movimientos sociales. Nuevos elementos fueron incluidos en la agenda de estudios del pensamiento latinoamericano: el concepto de centroperiferia, la categoría de dependencia, el colonialismo interno y la 
marginalidad como relación social y, al mismo tiempo, como consecuencia de la acumulación de capital en las sociedades dependientes.

Los conceptos de centro-periferia y de división internacional del trabajo serán centrales para estudiar la dinámica de acumulación del capitalismo que, para desarrollar las fuerzas productivas y fortalecer los procesos de industrialización en los centros económicos necesitaban de una periferia subdesarrollada, exportadora de materias primas. Es interesante observar que esta inserción dependiente y subordinada de América Latina en la economía mundial, que se inicia con la colonización europea, se mantiene después de la era colonial a partir de la reelaboración de mecanismos de la estructura de dominación colonial en un momento histórico poscolonial. Esta será una problemática profundizada por la teoría de la colonialidad y, principalmente, por la obra de Aníbal Quijano.

En sus estudios sobre democracia y socialismo, Theotonio muestra los límites del desarrollo capitalista dependiente. Afirma que el desarrollo capitalista en una situación de dependencia tenía una dinámica muy diferente a la que se había desarrollado en los países centrales, evidenciando una contradicción entre la acumulación capitalista dependiente y el proceso democrático. La acumulación en los países dependientes exigía tres elementos: una alta tasa de explotación de la fuerza de trabajo, una fuerte concentración económica y altos niveles de centralización del capital como mecanismo para compensar la exportación de la mayor parte del excedente económico hacia las economías centrales.

Esta lógica excluyente y marginalizadora necesitaba de un régimen político capaz de contener la reacción de los sectores sociales que afectaba y se revelaba incompatible con el crecimiento económico inclusivo y con una democratización política, bloqueando las expectativas de las burguesías nacionales y del proletariado emergente producto del desarrollo industrial. Apartir de este análisis Theotonio va a plantar el dilema entre fascismo y socialismo en los procesos políticos de América Latina. Fascismo que se confirmó con los golpes de Estado en Bolivia, Chile, Uruguay, Argentina y 
Brasil, y mostró las contradicciones intrínsecas del modelo económico que promovía, de profundo contenido antinacional.

Estas formulaciones y análisis se producen en el Centro de Estudios Socioeconómico (CESO), cuyos investigadores llegaron a ocupar cargos importantes en el gobierno de Salvador Allende. Entre ellos se destaca Orlando Caputo, que fue presidente de la Comisión Nacional del Cobre y uno de los directores de la empresa estatal del cobre, CODELCO. Se trata de un momento de enorme riqueza teórica y política, justamente por la articulación de ambas dimensiones: un enfoque que va adquiriendo creciente densidad teórica como matriz analítica de un proceso histórico concreto, el proceso chileno, y una coyuntura regional que va a inspirar y será la referencia permanente de esta construcción teórica.

En esos años Ruy Mauro Marini, que militaba en el Movimiento de Izquierda revolucionaria-MIR, escribe su libro clásico: Dialéctica de la Dependencia. Tal era la dinámica política del proceso chileno que para poder escribir este libro, Marini tuvo que confinarse por algunas semanas, sin paradero conocido, causando gran preocupación entre los amigos y compañeros de militancia. Grande fue la sorpresa cuando reapareció con uno de los libros más relevantes del pensamiento social latinoamericano bajo el brazo. Una de las características de esta escuela de pensamiento es, precisamente, su vínculo profundo a los procesos políticos concretos. La articulación entre teoría y praxis adquiere una importancia central en la obra de esta generación.

\section{Revolución científico-técnica y economía mundial}

Con el golpe de Estado de 1973 en Chile, el primer núcleo de la teoría de la dependencia se desarticula en una diáspora mundial. Después de casi seis meses de asilo en la Embajada de Panamá en Santiago, Theotonio fue uno de los últimos en conseguir el salvoconducto que le permitió viajar hacia la ciudad de México, donde fue rápidamente acogido como profesor de la Universidad Nacional Autónoma de México (UNAM). En el Instituto de Investigaciones Económicas, del cual fue director años más tarde, se reúnen 
también Vania Bambirra y Ruy Mauro Marini, lo que permitió continuar una fecunda colaboración intelectual que se expresó en una vasta producción teórica individual y colectiva. Por su tradición de política externa independiente en relación con Estados Unidos, México acogió a gran parte de los exiliados de las dictaduras militares en América Latina, convirtiéndose en un espacio privilegiado de circulación e intercambio de la intelectualidad de izquierda en la región, como lo fuera Chile en su momento.

Es en este período se desarrolla con más fuerza el tercer núcleo de preocupaciones teóricas en la obra de Theotonio, a través de un estudio sistemático de la Revolución Científico Técnica (RCT), como un aspecto central que va a marcar el desarrollo de las fuerzas productivas en nivel global. En el instituto de Investigaciones Económicas va a coordinar un grupo de estudios con alumnos de posgrado y jóvenes investigadores, entre los que desatacaba Leonel Corona, con quien estableció una estrecha colaboración, desarrollando un conjunto de actividades académicas como el Seminario de Ciencia y Tecnología de la División Superior de Economía de la UNAM, que se mantiene hasta el momento actual. Sus estudios sobre RCT se profundizarán en Japón, a inicios de los años de 1990, a partir de una invitación que recibe de la Universidad de Kioto para permanecer como profesor e investigador durante dos años en esta institución.

Los estudios de Theotonio analizan la RCT como un concepto que busca articular las transformaciones del desarrollo del capitalismo de manera integrada, a partir del papel radicalmente distinto que tiene el conocimiento científico en las actividades económicas. El capitalismo, como modo de producción, está fuertemente asociado a la transformación tecnológica, que se convierte en el elemento central del proceso productivo y la dinámica de acumulación. La revolución científico-técnica (RCT), que se introduce durante la Segunda Guerra Mundial, produjo una ruptura radical con el paradigma tecnológico de la Revolución Industrial del siglo XIX y la producción manufacturera.

El autor muestra que la ciencia deja de cumplir un papel secundario en la producción, para convertirse en el factor determinante en varias ramas de 
la industria que surgen después de la Segunda Guerra Mundial y que se van expandiendo al conjunto de la economía. La etapa de la RCT se caracteriza por una transformación profunda de las fuerzas productivas que se expresa en la automación o robotización de la producción que sustituye el trabajo humano por las máquinas; el uso de la electrónica como instrumento de transformación de los medios de producción y la posibilidad de producir fuentes de energía más poderosas, como la energía nuclear, que abren nuevas perspectivas energéticas en nivel global.

En esta nueva etapa la producción está cada vez más determinada por la invención e innovación y la planificación, exigiendo nuevos procedimientos de administración de las relaciones sociales. En consecuencia, se expanden las actividades de investigación y desarrollo en las empresas, como principal instrumento de competitividad. Al mismo tiempo, se amplían fuertemente las estructuras universitarias y de investigación para la producción de conocimiento y formación de científicos. El conocimiento pasa así a ocupar un lugar central de la economía y de la vida social, política y cultural.

El autor muestra que el desarrollo del capitalismo durante la segunda posguerra mundial estuvo fuertemente apoyado en la ampliación de la tasa de plusvalía a partir de dos elementos: la reducción salarial y la elevación de la tasa media de lucro que amplió las condiciones de sobre explotación de la mano de obra gracias a una fuerte intervención del Estado. El aumento de la participación estatal en la propiedad de sectores económicos de baja rentabilidad que transfieren su producción al sector privado a muy bajo costo favoreció los monopolios. Esta situación permitió una nueva onda de innovaciones apoyada en la aplicación de transformaciones tecnológicas acumuladas en el periodo de crisis y de guerra, lo que condujo a un aumento importante de la productividad en el período de posguerra.

Este proceso generó también una devaluación fuerte de los complejos industriales instalados antes de la guerra. La innovación crea nuevas estructuras industriales que absorben el avance tecnológico anterior acumulado y, al mismo tiempo, destruyen los complejos industriales que 
dependen de los ciclos tecnológicos anteriores y que entran rápidamente en obsolescencia, como lo mostró Joseph Schumpeter en sus análisis sobre la "destrucción creadora". La automación o robotización de la producción se desarrolla en la década de 1980 con el uso masivo de robots en Japón, posteriormente en Estados Unidos y, a partir de 1990, en las economías emergentes, particularmente China.

Los análisis de la RCT conducen a Dos Santos a un estudio más profundo del capitalismo de Estado asociado a la concentración tecnológica que produce $\mathrm{y}$, al mismo tiempo, como consecuencia de la concentración de la producción que tiende a asumir una forma espacial de grandes unidades de producción, en algunos periodos, y de pequeñas unidades integradas en amplios complejos industriales, en otros. Estos estudios muestran que la tendencia del capital es desconcentrar sus unidades productivas a través de holdings y otras formas empresariales, $\mathrm{y}$, al mismo tiempo, aumentar la concentración tecnológica, ya que las unidades productivas son interdependientes y forman parte de una misma unidad productiva. Esta complejidad creciente del sistema productivo y el aumento de la interdependencia entre unidades de producción y ramas de la economía promueve una concentración económica y empresarial aún mayor y, como consecuencia, también una mayor concentración del capital.

Esta centralización gigantesca del capital produce conglomerados de corporaciones multinacionales que necesitan de una creciente intervención del Estado para operar globalmente, garantizando una flexibilización financiera sin restricciones. Dos Santos muestra que el fortalecimiento del capitalismo de Estado es una consecuencia del crecimiento de la intervención del Estado en el proceso económico, contraponiendo la práctica económica al discurso doctrinario del libre mercado. Estas son las razones que ofrece el autor:

El proceso de concentración de la producción lleva a una composición orgánica del capital creciente, lo que produce una caída sistemática de la tasa de ganancia en los sectores de mayor concentración económica. En este contexto, para mantener una elevada tasa media de ganancia, el capital 
privado busca transferir progresivamente al Estado las actividades económicas menos lucrativas y servicios que los asalariados consumen, como salud, educación o habitación.

La expansión de las unidades de producción, que generan mayor concentración y centralización. Este fenómeno necesita de una creciente intervención del Estado para administrar el intercambio, la circulación y el propio proceso productivo, que violan sistemáticamente las leyes de mercado a través de precios regulados, subsidios estatales o tasas de interés artificiales. Los monopolios quiebran el funcionamiento normal del mercado, exigiendo la intervención del Estado para regular la economía.

La internacionalización de la producción, que se intensifica con la RCT, genera altos costos que dependen cada vez más del Estado. Estos gastos se concentran, en las primeras fases del capitalismo de Estado, principalmente en gasto militar, gastos diplomáticos y organización del aparato burocrático colonial y de "cooperación".

Este núcleo teórico condujo a una profundización de sus estudios sobre la economía mundial y del neoliberalismo como doctrina y como práctica económica. Sus análisis van a alcanzar un altísimo nivel de formulación teórica en su libro Del terror a la esperanza, auge y decadencia del neoliberalismo que tendrá continuidad y se ampliará en Desarrollo y civilización, donde ofrece una síntesis importante:

El neoliberalismo es un movimiento ideológico reaccionario (propio de las fases de recesión económica global de los ciclos de Kondratiev, como veremos posteriormente) que pretende detener las transformaciones socioeconómicas derivadas de las fases de crecimiento y avances globales, identificados por Kondratiev, propias del funcionamiento del capitalismo contemporáneo. Este movimiento de presión social e incorporación de reformas políticas está condicionado por varios factores:

La tendencia a la caída de la tasa de ganancia, originada por la importancia creciente de los gastos en maquinaria y materias primas (capital constante) que acompaña las innovaciones económicas (aumento de la composición orgánica del capital) y causada también por el aumento de la capacidad del trabajo asalariado para obtener mejores condiciones de remuneración, como consecuencia del pleno empleo generados en períodos de auge económico. Esta tendencia lleva al capital a buscar (neutralizar) las contra tendencias que pueden conducir a formas de "socialización de la propiedad privada", que llevan a expandir el monopolio y el capitalismo de Estado. Instrumentos privilegiados para neutralizar, en parte, la caída de la tasa de ganancia, a través de varios mecanismos de transferencia de valor. 
La implantación de la Revolución Científico Técnica en la década de 1940, estimulada por las ventajas ofrecidas por la aplicación de las innovaciones tecnológicas en la competencia capitalista. Ésta, sin embargo, es productora de una lógica propia, que provoca un impacto radical sobre las innovaciones tecnológicas y el funcionamiento del capitalismo, en el sentido de reducir el tiempo de trabajo socialmente necesario para la producción de mercancías, disminuyendo drásticamente las posibilidades de la ley del valor y de las relaciones mercantiles puras con el avance del capitalismo monopólico de Estado (DOS SANTOS, 2016).

La dimensión contradictoria del neoliberalismo, según Theotonio, radica en la capacidad que este tiene de detener las transformaciones económicas que se desprenden de la propia dinámica del capitalismo, a partir de un conjunto de reformas políticas que fortalecen el capitalismo monopólico de Estado. En varios trabajos sobre el tema el autor mostrará las contradicciones entre la doctrina neoliberal, que predica la disminución del papel del Estado en la economía y su práctica económica y política real, que fortalece la intervención del Estado para beneficiar la concentración del capital, los oligopolios y monopolios.

Estos análisis ofrecen un instrumental teórico poderoso para comprender la dinámica de la economía mundial del siglo XXI. Inclusive la emergencia China en el sistema mundial y las nuevas condiciones de la disputa científico-tecnológica, a partir de centralidad de la plusvalía relativa en el proceso de modernización de las fuerzas productivas que permiten el aumento de la productividad del trabajo de una manera tan colosal como la que estamos observando en Asia y particularmente en China. Hasta hace algunos años era relativamente común escuchar en los debates académicos argumentos que atribuían el gran crecimiento económico e industrial del modelo chino a la existencia de mano de obra esclava en ese país. Hoy en día nadie más se atreve a defender esta tesis.

La disputa por la innovación científica y tecnológica se presenta como una de las más implacables y va a marcar las nuevas tendencias del capitalismo contemporáneo en un contexto geopolítico de crecientes tensiones y de reorganización de intereses estratégicos, de territorios y de capacidades locales de producción científica en nivel planetario. 
De hecho, uno de los principales temas de estudio de Theotonio desde la década de 1980 fue entender en profundidad el proceso asiático: inicialmente Japón y luego China como grandes centros de la economía mundial, con una expresión fuerte en el sector financiero, que convierte a Shanghái en el centro financiero de Asia, con creciente capacidad de disputar con los grandes centros globales.

\section{Sistema mundial y proceso civilizatorio}

Un cuarto momento teórico en la obra de Theotonio es el estudio del desarrollo y del proceso civilizatorio como dimensiones que van a articular procesos históricos más generales. Tal vez la expresión más acabada de sus estudios sobre esta problemática se encuentra en su último libro, "Desarrollo y civilización: Homenaje a Celso Furtado", que es resultado de más de quince años de trabajo. En este libro, el autor articula, en un nivel de análisis más general, las múltiples determinaciones formuladas en sus estudios anteriores, que va a iluminar los procesos concretos a partir de una visión histórica de larga duración. Se trata de la obra de un intelectual maduro que piensa los procesos civilizatorios desde la perspectiva del tiempo milenario, retomando las formulaciones teóricas de la Escuela de los Annales y, particularmente, los aportes de Fernand Braudel:

Para situar correctamente la relación entre la teoría de la dependencia y la teoría del sistema mundial, debemos destacar, en primer lugar, el trabajo de Fernand Braudel, quien se proyectará, en las décadas de 1960 y 1970, como uno de los principales teóricos del sistema económico mundial, con su libro Civilización material: economía y capitalismo. Esta tradición puede verse como independiente del marxismo, a veces incluso como una crítica del mismo, pero construida, en gran medida, en el debate con este, en la relación dialéctica con el mismo y con sus diferentes formas: crítica del sectarismo en general, que involucra críticas al estalinismo, críticas de trotskistas, luxemburguistas, maoístas, titoistas, etc., que no lograron ofrecer una alternativa global a la altura de la fuerza de sus propuestas teóricas e históricas (DOS SANTOS, 2016, p. 115).

Braudel propone un análisis histórico en tres niveles, que implican duraciones diferentes: en la superficie, una historia episódica o de los acontecimientos, que se inscribe en el tiempo de corta duración. Es el tiempo breve que mide la vida de los individuos y la vida cotidiana, y puede ser la 
duración temporal más engañosa al mostrar los procesos históricos en sentido contrario a sus tendencias más generales. Con una profundidad media, el tiempo de la coyuntura, que tiene un ritmo más amplio y más lento. En un tercer nivel, el tiempo de la estructura, que determina siglos enteros.

El tiempo de la coyuntura surge a partir de la historia económica, que requería de un análisis que trascendiera la visión del tiempo del acontecimiento para pensar la economía como ciclos e inter-ciclo de diferentes duraciones, entre 50 y 70 años. Esto va a permitir analizar fenómenos más complejos, que necesitan de una cierta duración para desarrollarse plenamente. Por ejemplo, los ciclos hegemónicos formulados por Giovanni Arrighi, o los ciclos de la economía mundial, que tuvieron una de sus elaboraciones más importantes en la obra del economista ruso Nicolai Kondratiev.

El enfoque de los ciclos largos de la economía de Kondratiev, propone que la economía mundial se comporta en ciclos de 50 a 70 años de duración. Cada ciclo presenta una primera fase (A) donde predomina el crecimiento económico y recesiones económicas moderadas, seguida de una fase (B) caracterizada por una recesión económica con presencia de algunos periodos de moderado crecimiento. Ambas fases tienen una duración de 25 a 35 años. Es interesante destacar que es en la fase (B) de crisis económica que se van a producir las innovaciones tecnológicas más importantes, como necesidad inminente para superar la crisis y abrir nuevos ciclos de expansión económica, que abre paso a la fase (A) de un nuevo ciclo. Theotonio incorpora y profundiza el enfoque de Kondratiev en sus estudios sobre la economía mundial.

La tercera dimensión temporal que Braudel propone, el tiempo de la estructura, con una duración más larga, secular, va a permitir entender los procesos aparentemente invisibles pero que están presentes y que emergen a partir de una determinada correlación de fuerzas sociales o políticas. La idea de la larga duración en la comprensión de fenómenos estructurales conduce a una historia múltiple, en la que las civilizaciones cumplen un papel central. 
Theotonio recoge y reelabora este enfoque para analizar el proceso civilizatorio como proceso histórico de larguísima duración, del tiempo milenario, formulando así elementos fundamentales para entender los cambios en el sistema mundial y la geopolítica contemporánea. En sus análisis sobre la emergencia de China en la economía mundial, el autor identifica la reconstitución de un proceso civilizatorio de más de tres mil años de continuidad histórica, de acumulación política y desarrollo tecnológico, ya que fue el centro más dinámico de la economía mundial mucho antes del surgimiento del capitalismo y que reemerge a fines del siglo pasado como nuevo centro económico y potencia mundial.

El autor dialoga con las tesis de André Gunder Frank, que analiza el sistema mundial a través de un horizonte temporal milenario. La tesis principal de Frank es que el sistema mundial tiene una historia de, al menos, 5 mil años y que el ascenso y la posición predominante de Europa y occidente en este sistema mundial es solo un acontecimiento reciente.

El economista alemán establece un debate extremamente interesante con Immanuel Wallerstein y los demás formuladores de la teoría del sistemamundo. La principal crítica radicaba en la evaluación de que el sistema mundial en sí mismo se constituye mucho antes del advenimiento del capitalismo, y que tendría una duración de por lo menos 5 mil años y no 500 años como defienden los teóricos del sistema-mundo. Esta tesis de Frank se basa en tres puntos principales:

1. El sistema de acumulación del capital es una especie de fuerza motora de la historia del sistema mundial y no una diferencia específica del sistema mundial moderno, que emerge con el capitalismo.

2. Al mismo tiempo, la alternancia de liderazgos hegemónicos $y$ rivalidad por la hegemonía del sistema mundial no es un proceso exclusivo del sistema mundial desde 1492, sino que marca la historia del mundo desde mucho antes.

3. Los ciclos económicos largos de la economía mundial, con fases ascendentes (A) y descendentes (B), son una característica importante del moderno sistema mundial, cuyo proceso de acumulación cambia con la 
posición centro-periferia. Sin embargo, estos ciclos largos, se pueden observar mucho antes de 1492.

En 2003, la Cátedra y Red de la UNESCO y de la Universidad de Naciones Unidas sobre Economía Global y Desarrollo Sustentable, que Theotonio presidia desde su creación en 1997, organizó en Río de Janeiro un gran seminario para discutir el proceso de globalización y hegemonía mundial. En este cónclave participaron los referentes más importantes del pensamiento crítico mundial. Fueron más de 80 intelectuales alrededor de una mesa redonda debatiendo a lo largo cinco días, frente a un nutrido auditorio de jóvenes estudiantes, investigadores y académicos. Una de las primeras mesas de debate discutía el tema de globalización y sistema mundial, con la participación de Immanuel Wallerstein, Giovanni Arrighi, Samir Amin, André Gunder Frank y Theotonio. André había perdido la conexión en Nueva York, y tuvo que pasar una madrugada en el aeropuerto hasta el próximo vuelo a Río de Janeiro, ya con un cáncer avanzado en aquel momento. Aterrizó en Río poco antes del inicio de la mesa donde debía participar y pidió, desde el aeropuerto, que lo mantuvieran en la programación original. Para sorpresa de sus colegas, y de todo el público presente, llegó a la sala de reuniones con su maleta en mano y tomó inmediatamente su lugar. Escuchó atentamente todas las presentaciones y, llegado su turno, inició su intervención con el estilo polémico que lo caracterizaba. Hizo una crítica contundente, principalmente a Wallerstein y Arrighi que defendían la idea de que el sistema-mundo surge en el siglo XV con la emergencia del capitalismo y sus formas de acumulación, y que se va consolidando con gran éxito hasta convertirse en el modo de producción dominante y articulador de la civilización occidental.

Para Frank esa visión, a pesar de crítica, no conseguía liberarse de una influencia eurocéntrica, al negar la existencia de sistemas mundiales anteriores al capitalismo y a la emergencia de occidente como centro del sistema mundial. Después de una dura crítica formulada a los amigos y sustentada con vehemencia, Frank reconoció que Giovanni Arrighi, al escribir 
Adam Smith en Pekín, "se había reivindicado de su postura eurocéntrica" pues estaba aceptando la existencia del sistema mundial antes del capitalismo.

Este seminario fue el último en el que confluyeron los principales formuladores de la teoría del sistema mundial. Fue un encuentro histórico. Tanto por el contenido, que mostró el vigor del pensamiento crítico mundial y su capacidad para entender las tendencias de la coyuntura que luego se verificarían en los procesos concretos, como también por el debate abierto y sincero que caracteriza un pensamiento en permanente reelaboración y resignificación... Estas discusiones mostraron la potencia del debate de ideas y la polémica como instrumentos de construcción de conocimiento y están recogidas en un libro de cuatro volúmenes publicado en Brasil bajo el título de Os impasses da globalização (DOS SANTOS et al., 2003-2005).

Este marco interpretativo, que Theotonio va a compartir y contribuir a formular, permite entender cómo China, en poco más de 30 años, deja de ser una economía campesina para convertirse en la mayor economía del mundo, como lo muestra el ranking de las mayores economías del mundo en 2014, cuando China desplaza a Estados Unidos y se coloca en primer lugar en tamaño del PIB en dólar ajustado a su poder de compra local (PPP). Es poco útil analizar la emergencia de China como un fenómeno reciente. Tal vez la forma más apropiada sea entenderla como la reemergencia de un proceso civilizatorio milenario, que ya fue un imperio-mundo y el centro de la economía mundial durante casi siete siglos (s. XI a XVII), donde se producía la manufactura más elaborada que se exportaba hacia Europa para abastecer el consumo suntuoso de las monarquías y la aristocracia. Durante este periodo China tuvo un superávit comercial muy grande, convirtiéndose en receptora de prácticamente todo el oro y la plata del sistema mundial euroasiático, al que se suma el continente americano a partir de la colonización europea, principalmente como exportadora de minerales preciosos. En este período, en que China era el centro del sistema mundial, Europa ocupaba un lugar periférico, sin condiciones económicas ni políticas de asumir un liderazgo hasta inicios del siglo XVIII, cuando surgen las 
primeras revoluciones industriales y el Estado moderno europeo como modelo político.

Este enfoque muestra la articulación entre desarrollo y proceso civilizatorio. De ahí que la forma más pertinente de entender el fenómeno chino contemporáneo sea verificar que se trata de la rearticulación de un conjunto de capacidades económicas, políticas y tecnológicas que forman parte de un legado histórico de más de 3 mil años y casi 700 años de centralidad económica. Es decir, de un proceso civilizatorio de larguísima duración que se reconfigura entre finales del siglo XX e inicios de siglo XXI, lo que explicaría la velocidad de las transformaciones de la economía china y de sus capacidades de conducir con creciente centralidad el sistema mundial.

La dialéctica de duraciones del tiempo histórico y la dimensión del tiempo milenario, o tiempo de la civilización, aparecen como elementos fuertes en la reapropiación que hace la obra de Theotonio para entender los procesos contemporáneos en pleno desarrollo. Mucho antes que sus manifestaciones más explícitas, el autor prevé el fortalecimiento de las economías emergentes en el panorama mundial, y el surgimiento de los BRICS como espacio de articulación de estas nuevas fuerzas. En 2004, en un seminario que organizó en Río de Janeiro, propuso la creación de una red de colaboración académica entre Brasil, Rusia, India, China y África del Sur (BRICAS), coordinada por la Cátedra y Red UNESCO sobre Economía Global y Desarrollo Sustentable-REGGEN, que él dirigía. Esta iniciativa contó con la participación de un conjunto de instituciones académicas y de investigación de los cinco países, entre las que destacaban la Academia China de Ciencias Sociales, la Universidad de Delhi, el Centro de Estudios de los Países en Desarrollo de la India, la Academia Rusa de Ciencias, el Congreso Sudafricano y, en Brasil, la Universidad Federal de Río de Janeiro, la Universidad Cándido Mendes y la Universidad Federal Fluminense, cuyo rector propuso crear la sede de esta red en su campus de Niteroi. Varios académicos se sumaron a la coordinación de esta iniciativa: Gao Xian, profesor senior de la academia China de Ciencias Sociales; Xie Shou-guan y Huan Ping, de la misma institución; Manoranjan Mohanty, director del Centro de Investigación de los 
Países en Desarrollo; Deepak Nayyar, vicerrector de la Universidad de Delhi, Valdimir Davydov de la Academia Rusa de Ciencias, entre muchos otros académicos a los que este proyecto fue movilizando. Hoy en día, en que la aproximación con Asia se ha intensificado, esta iniciativa no sería novedosa. Sin embargo, cuando fue propuesta, representaba un proyecto pionero en América Latina, un esfuerzo por potenciar la cooperación académica, científica y tecnológica entre los países del Sur y, particularmente, entre las instituciones académicas de las potencias emergentes.

En sus análisis, Theotonio muestra que el fortalecimiento del mercado interno chino, que tiende a crecer acompañado de una valorización permanente del Yuan, continuará presionando, a través de su creciente demanda, la oferta mundial. No solo de materias primas, lo que impacta directamente a América Latina, sino también sobre sectores industriales y de servicios, que afecta a EUA, Europa, Japón, Corea del Sur, Rusia y gran parte de Asia. La demanda china articula un gran polo de desarrollo apoyada en la expansión y universalización de patrones tecnológicos que integran las innovaciones tecnológicas de la década de 1980: robotización e inteligencia artificial, nuevos materiales y biotecnología. Estos análisis, recogidos en su último libro "Desarrollo y civilización", son anteriores al lanzamiento del proyecto chino de la Nueva Ruta de la Seda, en setiembre de 2013. Sin embargo, ya capturaban las tendencias del proceso asiático $\mathrm{y}$, particularmente, la centralidad de China en la reconfiguración del continente euroasiático y de la economía mundial en las próximas décadas.

Estas investigaciones lo conducían a un nuevo proyecto teórico, que sería un estudio detallado del capitalismo de Estado en el mundo contemporáneo, a partir de la experiencia china. Proyecto que no llegó a realizar, y que seguramente quedará como una agenda de investigación para las nuevas generaciones.

Regresando al análisis, podemos afirmar que China ya no está promoviendo incipientemente la transferencia tecnológica o integrando las innovaciones tecnológicas de la década de 1980, sino disputando tecnología de punta en sectores estratégicos, que incluyen la nanotecnología, 
producción de nuevos materiales para nuevos ciclos tecnológicos e industriales, energías renovables, e inclusive grandes avances tecnológicos en el parque industrial militar. China tuvo un aumento importante en su participación en el gasto militar mundial. Pasó de 2\% del gasto mundial en 2010 a $13 \%$ en 2019. Mientras que Estados Unidos cae de $50 \%$ a 35\% en el mismo periodo, según el SIPRI. Es decir, en este momento China representa el segundo gasto militar mundial y está desarrollando una significativa colaboración tecnológica con Rusia, que ya fue una potencia militar durante el periodo de la Guerra Fría.

Esto marca un nuevo momento en que China está disputando la centralidad tecnológica inclusive en el parque industrial militar, desafiando una vez más a Estados Unidos, en un mundo donde todos los indicadores muestran un desplazamiento del dinamismo económico desde Occidente hacia Oriente y desde el Norte hacia el Sur.

Los estudios de Theotonio sobre desarrollo y proceso civilizatorio permiten comprender mejor la nueva geopolítica mundial, que no se restringe a disputas comerciales, económicas, científicas o tecnológicas, sino que tienen que ver, principalmente, con una fuerte disputa de visones de mundo. En última instancia, la reemergencia de China en el sistema mundial refleja la emergencia de una visión asiática del mundo.

¿Cuál es el papel de América Latina? ¿Cuál es el papel de la región que se inserta de manera dependiente y subordinada en el sistema mundial desde finales del siglo XV cuando se inicia el periodo colonial en el continente? Situación que se reelabora a lo largo de los últimos cinco siglos, inclusive después de las guerras de independencia de las colonias españolas. Es en este punto que la obra de Theotonio va a recuperar la profundidad del proceso civilizatorio americano a través de la civilización Caral, que surge en la costa norte del Perú, en la región de Supe. Descubierta hace más de 20 años por la arqueóloga Ruth Shady, la civilización Caral, con 5 mil años de antigüedad, convirtió el proceso civilizatorio americano en uno de los más antiguos del planeta, solo después de Egipto y Mesopotamia. 
Esta sociedad altamente desarrollada constituye el antecedente más antiguo del proceso civilizatorio americano y tal vez el primer momento de integración del continente a partir del intenso intercambio comercial, cultural y lingüístico (fue el periodo de formación de las lenguas madres del continente) que se extiende desde la costa norte del Perú hacia la región andina, amazónica e inclusive América Central, según algunas hipótesis más recientes.

Desarrolló una importante producción agrícola a partir de conocimientos en ingeniería agraria que permitieron la construcción de un sofisticado sistema de riego, reservorios de agua y manejo genético de semillas para mejorar la calidad de los cultivos. Fue un gran productor de algodón y desarrolló una amplia red comercial de tejidos de algodón y redes de pesca. Además, fue una civilización capaz de desenvolver importantes innovaciones tecnológicas, como un sistema de construcción antisísmica que posibilitó que sus pirámides, tan antiguas como las de Egipto, permanecieran intactas a pesar de localizarse en una región altamente sísmica.

Estos son elementos que Theotonio va a estudiar y revisitar en un esfuerzo teórico que buscaba una comprensión más profunda de los procesos de integración que emergieron a inicios del siglo XXI y que constituyen proyectos históricos de larga duración. Va a formular la idea de una civilización planetaria, como un nuevo marco histórico del siglo XXI, capaz de abrir camino hacia una nueva convivencia humana universal. El redescubrimiento del mundo no europeo, que se realizó en Asia, África y América Latina, coloca el desafío de una reelaboración histórica de gran envergadura, capaz de superar la visión eurocéntrica y recoger los aportes de los diferentes procesos civilizatorios de la humanidad.

\section{La perspectiva teórico-metodológica: teoría y praxis}

Desde el punto de vista teórico metodológico, la obra de Theotonio es una expresión de un movimiento intelectual más amplio en América Latina. El pensamiento crítico latinoamericano va a contribuir de diferentes maneras a la comprensión de los procesos históricos concretos de la región, 
pero también al desarrollo y profundización de matrices teóricas más generales y a la comprensión del sistema mundial como momento histórico totalizador, dialéctico y complejo. Desde luego, no se trata de simples transposiciones conceptuales, sino de procesos de apropiación y reelaboración de marcos teóricos más generales para entender procesos históricos concretos. La obra del sociólogo brasileño Guerreiro Ramos, autor de La reducción sociológica, muestra este movimiento de apropiación de matrices teóricas generales para entender la realidad social brasileña. Apropiación que significa, necesariamente, una reelaboración teórica y conceptual. La "reducción sociológica” para Guerreiro Ramos, representa un proceso de "nacionalización" de matrices teóricas más generales, nacionalización de la sociología europea y de Estados Unidos para colocarlas en capacidad de comprender la realidad brasileña.

Theotonio fue, desde muy joven, un estudioso de Guerreiro Ramos. En agosto de 1958, con 22 años, publicó un artículo titulado "Perspectivas de la reducción sociológica" analizando el libro clásico de este autor, La reducción sociológica, destacándola como una obra fundamental para entender las ciencias sociales en Brasil.

Para Guerreiro Ramos, la sociedad brasileña condiciona el pensamiento brasileño y este pensamiento solo se torna crítico cuando asume metódicamente la conciencia de esta sociedad y de su proceso histórico. Este concepto es fundamental y por eso él coloca entre las leyes de la reducción sociológica la ley de las fases, y es a través del proceso histórico que podemos percibir que para poseer una teoría de la sociedad brasileña es necesario, al mismo tiempo, una teoría del mundo donde está inserta esta sociedad [...] (DOS SANTOS, 1958).

Por otro lado, la idea proceso civilizatorio como núcleo analítico va a encontrar en el concepto de "eurocentrismo" un instrumento poderoso para explicar procesos de dominación política, económica y cultural. Los sistemas de dominación colonial se apoyaron en estructuras sociales y productivas desarrolladas por los pueblos indígenas de la región andina y de América Central, exactamente donde se concentraron las grandes civilizaciones del continente americano. El proceso colonizador y las estructuras de dominación impuestas por éste invisibilizaron los grandes avances de los pueblos originarios. 
Esa densa obra intelectual se desarrolló en un dialogo intenso con el pensamiento crítico mundial, con cuya intelectualidad convivió desde muy joven, dado que colaboró con centros académicos y de investigación en todo el mundo, participó de debates, conferencias, seminarios, redes académicas, y también como profesor visitante durante periodos más largos.

Ciertamente Theotonio no fue un intelectual de laboratorio, sino todo lo contrario. Se trata de la obra de un intelectual orgánico que tuvo la capacidad de articular una densa y amplia producción científica a los procesos de transformación social y política y a las necesidades de la dinámica de lucha de clases, colocándose al servicio de los procesos de transformación social. Su trabajo intelectual estuvo acompañado de una intensa militancia política. En 1961 funda la Organización Revolucionaria Marxista Política Operaria (POLOP) que integraba a militantes de la Juventud Laborista de Minas Gerais, la Juventud Socialista de Río de Janeiro, San Pablo y Bahía, y algunos otros grupos políticos como la Liga Socialista de San Pablo. Esta organización expresaba una crítica radical al estalinismo y a la visión soviética del marxismo, recuperando las experiencias políticas que marcaron la década de 1950, como el movimiento de países del Tercer Mundo y la Revolución cubana que impactó profundamente a la izquierda latinoamericana. En 1964 asume la dirección nacional de esta organización, cuando ocurre el golpe militar en Brasil, que lo condena a 15 años de prisión por el delito de "mentor intelectual de la penetración subversiva en el campo".

En 1966, después de dos años de clandestinidad en San Pablo, llega exiliado a Chile donde permaneció 8 años en intensa colaboración con las luchas políticas que llevaron al gobierno a la Unidad Popular. El golpe de Estado contra Salvador Allende en 1973 lo obliga a un segundo exilio en México, al colocarlo en los primeros lugares de las listas negras de la dictadura que lo buscaba "vivo o muerto". En 1979 funda el Partido Democrático Trabalhista (PDT), del cual será dirigente nacional, junto a Leonel Brizola y algunos de los más destacados pensadores sociales brasileños, como Darcy Ribeiro, Luiz Alberto Moniz Bandeira, Paul Singer y 
Vania Bambirra. En 1982 participa como candidato por el PDT a Gobernador del estado de Minas Gerais y en 1986 como candidato a Diputado Federal por el mismo estado. Pierde ambas elecciones. En 1999 acepta la invitación de Anthony Garotinho, Gobernador del estado de Río de Janeiro, al cargo de Secretario de Relaciones Internacionales, función que mantiene durante cuatro años. La militancia y actividad política van a acompañar su intensa producción intelectual.

En los casi veinte años de convivencia lo vi dividirse entre las actividades académicas, la orientación a sus alumnos, la participación en espacios políticos y de militancia, la colaboración con artículos de opinión en columnas quincenales en varios periódicos latinoamericanos, los infinitos viajes alrededor del mundo para participar de seminarios, conferencias, mesas redondas y una multiplicidad de reuniones científicas y académicas. Siempre me llamó la atención la energía, que a veces parecía inagotable, desplegada en cada una y todas estas actividades, el entusiasmo con el que abrazaba cada nuevo proyecto y la generosidad con la que se dedicaba a sus alumnos y colaboradores. Lo veía escribir a cualquier hora del día o de la madrugada, en su propia sala de trabajo en casa, en las cabinas de avión, en los pasadizos de los aeropuertos o en los "bussiness centers" de los hoteles en cualquier ciudad del mundo. Siempre se llevó muy mal con los computadores portátiles, a pesar de vanagloriarse de haber sido uno de los primeros usuarios de laptop de su generación.

La militancia y participación en los procesos políticos concretos y su labor proficua como intelectual y pensador son dos dimensiones inseparables en su obra. Dimensiones que se articulan y retroalimenta de manera profunda, dejando un legado de teoría y praxis a las nuevas generaciones.

\section{Sobre la organización de esta obra}

No fue una tarea fácil realizar esta selección. En primer lugar, debido a la amplitud de la obra científica, con 34 libros publicados en varias ediciones traducidas a 18 idiomas y más de un centenar de artículos científicos y 
capítulos de libros. Para que esta antología fuera representativa de la obra fue necesario superar ampliamente la extensión promedio de CLACSO para esta colección, que hoy se entrega en dos volúmenes y casi 2 mil páginas. Además, como lo señala Francisco López Segrera en la introducción al segundo volumen, se hizo necesario identificar la versión más completa de cada libro seleccionado, que con cada edición sufría actualizaciones y, eventualmente, se enriquecía con nuevos análisis.

La segunda dificultad se presentó en pleno trabajo de selección y preparación, cuando Theotonio, que participó activamente en el proceso junto a los organizadores, descubrió una enfermedad muy grave que le quitó la vida algunos meses después, en febrero de 2018. La fase final de selección tuvo que realizarse entre largas jornadas hospitalarias y el poco tiempo y energías que dejaba disponible el complejo tratamiento médico que mantuvo por varios meses, lo que impidió avanzar conforme se había planeado inicialmente.

En la selección de textos, se dio preferencia a los temas teóricos de fondo en relación con los análisis empíricos referidos a períodos o casos concretos, reduciendo estos últimos a lo estrictamente necesario para mostrar la dinámica histórica concreta de los análisis teóricos más generales. A pesar de que se trató de evitar desmembrar algunos libros claves que fueron escritos de manera muy orgánica, el lector encontrará en esta publicación partes o capítulos del conjunto de libros que componen la obra completa. Para dar mayor articulación y fluidez a la antología, se prefirió retirar algunos libros cuyo contenido temático está parcialmente recogido en la selección, dando prioridad a los temas más generales y un menor énfasis a los análisis más específicos de coyuntura.

Los textos seleccionados en cada una de las cuatro partes que componen la antología no obedecen a un orden cronológico, sino a una organización temática.

La Primera parte: Cuestiones de método reúne los escritos teóricos y metodológicos más generales en torno a algunos conceptos claves como clase social, fuerzas productivas y relaciones de producción, el concepto de 
revolución científico-técnica y su impacto en el proceso de valorización y acumulación de capital.

La Segunda parte: La dimensión tecnológica de la crisis internacional recoge los principales trabajos sobre economía mundial, crisis internacional y sus efectos en el Tercer Mundo y en el orden económico y estructura de poder mundial. También reúne los trabajos sobre dependencia tecnológica y las condiciones para una estrategia científico-tecnológica de la región.

En la Tercera parte: La crisis internacional y la estructura del poder mundial el lector encontrará trabajos más recientes sobre el proceso de integración latinoamericana, así como un amplio estudio sobre el neoliberalismo y sus contradicciones como doctrina y como proyecto económico y político, que el autor profundiza en el libro Del terror a la esperanza, auge y decadencia del neoliberalismo.

La Cuarta parte: Desarrollo, democracia y socialismo concentra principalmente los análisis sobre dependencia, democracia y socialismo, tanto desde un enfoque teórico-metodológico como desde el análisis de la praxis política de los procesos históricos concretos. Algunos libros clásicos de la obra del autor, como Socialismo o fascismo: el nuevo carácter de la dependencia y el dilema latinoamericano o Democracia y socialismo en el capitalismo dependientes están recogidos en esta sección. Se incluyó también parte del libro Bendita crisis que ofrece un análisis detallado de la experiencia chilena durante el gobierno de Salvador Allende a partir de un conjunto de artículos escritos entre 1971 y 1973, al calor de la lucha política en Chile. Para completar este panorama de análisis político, se incluyeron también algunos capítulos del libro El camino brasileño hacia el socialismo que profundiza el estudio del proceso brasileño y propone un programa político de transición al socialismo.

La Quinta parte: Sistema mundial, imperialismo y dependencia reúne los trabajos sobre el debate conceptual en torno a la dependencia, el imperialismo contemporáneo y sus contradicciones económicas y políticas desarrollados en los libros Imperialismo y dependencia y Teoría de la 
dependencia: balance y perspectivas. Esta sección ofrece también las reflexiones sobre el encuentro teórico entre la teoría de la dependencia y la teoría del sistema mundial, así como sobre el proceso civilizatorio, abordado en su último libro Desarrollo y civilización. Un homenaje a Celso Furtado.

Como el lector podrá observar, esta antología, a pesar de ser extensa, recoge apenas lo indispensable de la producción científica del autor. Quien pretenda profundizar en su estudio puede encontrar un vigoroso acervo analítico en las Obras reunidas de Theotonio Dos Santos, publicadas por el Instituto de Investigaciones Económicas de la Universidad Nacional Autónoma de México-UNAM en 2015, bajo la coordinación de Carmen del Valle y Javier Jasso. Este acervo está disponible en el repositorio de la página web de esta universidad. ${ }^{4}$

Estamos convencidos de que esta antología constituye un aporte de gran valor para los estudiosos y académicos, pero también para quienes están en la lucha política cotidiana. Representa la recuperación de un legado vigoroso del pensamiento crítico latinoamericano que, por su originalidad, profundidad y osadía, se convierten en un instrumento de análisis para comprender la dinámica de capitalismo contemporáneo y los desafíos de América Latina en el horizonte histórico del siglo XXI.

Finalmente, quisiera expresar mi profundo agradecimiento al Consejo Latinoamericano de Ciencias Sociales por el compromiso con este proyecto y por el enorme trabajo desplegado para hacer posible la publicación de esta obra. Agradezco particularmente a Pablo Gentili, que propuso la idea durante la XXV Asamblea general de CLACSO en 2015; a Karina Batthyány, que le dio continuidad; a Nicolás Arata y Lucas Sablich que desde el área editorial acompañaron con enorme entusiasmo y dedicación cada detalle de la edición, a Estevão Musa que colaboró con la preparación de los materiales para este prólogo y a Iván Bambirra por la lectura crítica de los textos. Un

\footnotetext{
${ }^{4}$ En el momento en que escribimos este texto, el enlace de acceso a las obras reunidas es http://ru.iiec.unam.mx/id/eprint/4086
} 
agradecimiento especial a Francisco López Segrera por haber compartido conmigo esta jornada y el desafío de organizar esta antología.

Rio de Janeiro, noviembre de 2020

\section{Referencias}

DOS SANTOS, Theotonio [Theonio Junior]. Oferendas: Um poema-oração para Yemanjá e para a humanidade inteira. Inédito. (s.f.).

DOS SANTOS, Theotonio [Theonio Junior]. Che, ópera pop latinoamericana. Mimeo. (s.f.)

DOS SANTOS, Theotonio [Theonio Junior]. A Construção. Belo Horizonte: Ediciones Complemento, 1957.

DOS SANTOS, Theotonio [Theonio Junior]. Perspectivas de la Reducción sociológica. Diario de Minas, 24 de agosto, 1958.

DOS SANTOS, Theotonio. Quais são os inimigos do povo? Rio de Janeiro: Civilização Brasileira, 1962.

DOS SANTOS, Theotonio. 1970. El concepto de clases sociales. Santiago de Chile: Prensa Latinoamericana. [Ediciones en español Buenos Aires: Galerna, 1973; Caracas: Fundación Editorial el perro y la rana, 2007. Edición en portugués Petrópolis: Vozes, 1985].

DOS SANTOS, Theotonio. 1973/2003. Teoría de la dependencia: balance y perspectivas. Buenos Aires: Editora Sudamericana. [Edición en mandarín Beijing: Editora de la Academia China de Ciencias Sociales. Edición en portugués Río de Janeiro: Civilização Brasileira, 2000. Edición en español México: Plaza \& Janés 2002].

DOS SANTOS, Theotonio. Para Iugoslávia: uma tardia manifestação de amor. Mineo, 2002.

DOS SANTOS, Theotonio, 2004. Do terror à esperança: auge e declínio do neoliberalismo [Título en español Del terror a la esperanza, auge y decadencia del neoliberalismo]. Aparecida: Ideias \& Letras. [Edición en mandarín Beijing: Academia China de Ciencias Sociales, 2003. Edición en español Caracas: Monte Ávila, 2007].

DOS SANTOS, Theotonio. Desarrollo y civilización. Homenaje a Celso Furtado. [Título en portugués Desenvolvimento e civilização: homenagem a Celso Furtado]. Río de Janeiro; Buenos Aires: UERJ; CLACSO, 2016. 
DOS SANTOS, Theotonio; DEL VALLE, Carmen y JASSO, Javier (Coords.).

Obras reunidas de Theotonio dos Santos. México: Instituto de Investigaciones Económicas, UNAM, 2015.

DOS SANTOS, Theotonio; MARTINS, Carlos Eduardo; SÁ, Fernando;

BRUCKMANN, Mónica. Os Impasses da globalização, 4 Vol. Río de Janeiro:

PUC-Rio; Loyola, 2003-2005.

DOS SANTOS, Theotonio. Memorial. Niterói, RJ: UFF, 1994. 